\title{
Die meritokratische Neugestaltung der österreichischen Integrationspolitik zwischen Rhetorik und Policy
}

\author{
Oliver Gruber ${ }^{1,}$, Astrid Mattes ${ }^{1}$, Jeremias Stadlmair ${ }^{1}$ \\ 1 Institut für Politikwissenschaft, Universität Wien, Austria \\ * E-Mail: oliver.gruber@univie.ac.at
}

\section{Zusammenfassung}

Waren politische Debatten zur Integration von MigrantInnen lange Zeit durch einen Fokus auf Kultur und Werte gekennzeichnet, gewinnen „leistungsorientierte“ Zugänge zunehmend an Relevanz. Die österreichische Integrationspolitik nach der Einführung eines Staatssekretariats für Integration (2OII-20I3) zeigt anschaulich, wie ein solcher Zugang zum Leitprinzip einer Regierungspolitik werden kann. Der Beitrag geht dem Ansatz „Integration durch Leistung“ in einer Analyse von Rhetorik und Policy nach. Er untersucht einerseits das Leistungsnarrativ und sein Verhältnis zum bisherigen Integrationsdiskurs und stellt aufbauend darauf dar, ob bzw. wie diese diskursive Neuausrichtung in Policies übersetzt wurde. Dabei zeigt sich, dass sich der Ansatz zentral auf traditionelle Leistungskriterien der Arbeitsmarkt- und Bildungspolitik stützt, darüber hinaus aber nur partielle Erweiterungen des Leistungsbegriffs beinhaltet. Diese Gegenüberstellung erlaubt schließlich, immanente Ambivalenzen eines Leistungskonzeptes für die Integration von MigrantInnen aber auch seine Inklusionsund Exklusionspotentiale zu diskutieren.

\section{Schlüsselwörter}

Integrationspolitik, Meritokratie, Diskurs, Policyanalyse, Österreich

\section{Immigrant integration and meritocracy in Austria}

\begin{abstract}
While the political debate on the integration of immigrants has traditionally been tied to cultural aspects, „meritocratic“ arguments have increasingly gained relevance. With the introduction of a „State Secretariat for Integration“(20II-2OI3), Austrian politics on immigrant integration have turned into a prototype of this reorientation. This article studies the meritocratic approach to integration by the Austrian government in a two-stage analysis. Firstly it analyses the government's new policy narrative and its relation to previous rhetoric on immigrant integration. Secondly it demonstrates, if and how this discourse has been translated into policies. It demonstrates that the meritocratic approach largely relies on criteria derived from other policy areas, most importantly labor market and education, whereas its extension to other forms of action remains limited and inconclusive. The findings underline the intrinsic ambivalences of the meritocratic logic if applied to immigration integration policy, yet they also allow for a critical assessment of its inclusive and exclusive potential.
\end{abstract}

\section{Keywords}

Immigrant integration, meritocracy, discourse analysis, policy analysis, Austria

The authors have declared that no competing interests exist. 


\section{Einleitung ${ }^{1}$}

In ganz Europa werden „Migration und Integration“ kontroversiell diskutiert. Während nationale Debatten lange Zeit durch einen Fokus auf Kultur und Werte gekennzeichnet waren, bekommen leistungsorientierte Integrationsansätze in den EU-Mitgliedsstaaten zunehmende Relevanz. Mit dem Ausbau von „civic integration policies“, meist verpflichtenden Sprach- und Staatsbürgerschaftskursen bzw. -tests (Goodman 20IO; Joppke 2007), wuchs das Bestreben, Kriterien für den Integrationsgrad von MigrantInnen zu bestimmen, und damit "Integrationsleistungen“ gleichermaßen förderbar wie einforderbar zu machen (Gruber/ Mattes 2015; Kunz 20II). War der Leistungsanspruch häufig eher implizit enthalten, so zeigt die österreichische Integrationspolitik seit der Einrichtung eines Staatssekretariats für Integration (im folgenden SSI genannt) - und damit der erstmaligen Institutionalisierung des Politikfeldes auf bundespolitischer Ebene - anschaulich, wie „Integration durch Leistung" zum plakativen Leitprinzip einer Integrationsprogrammatik werden kann.

Der Beitrag nimmt dies zum Anlass, dem meritokratischen Ansatz in der Gestaltung der österreichischen Integrationspolitik der Bundesregierung nachzugehen. Er tut dies zunächst mit einer Diskussion des Meritokratiekonzeptes (2.I) sowie einer kursorischen Entwicklungsgeschichte der österreichischen Integrationspolitik (2.2), bevor das methodische Design der Analyse dargestellt wird (3). Der empirische Teil geht anschließend der Frage nach, wie der meritokratische Anspruch im politischen Diskurs zum Ausdruck kommt (4.I), ob bzw. wie diese diskursive Neuausrichtung auch in entsprechende Maßnahmen (Policies) übersetzt wurde (4.2) bzw. welche Kontinuitäten und Brüche zwischen beiden Ebenen bestehen (4.3). Dies erlaubt schließlich eine kritische Einschätzung der immanenten Ambivalenzen eines Leistungskonzeptes für die Integration von MigrantInnen sowie eine Gegenüberstellung seiner Inklusions- und Exklusionspotenziale (5.).

\section{Leistung als gesellschaftliches Integrations- konzept?}

\subsection{Meritokratische Ordnungen von Gesellschaft}

Meritokratie bezeichnet sozialwissenschaftlich ein politisches und soziales Gestaltungsprinzip, in welchem Leistungen als maßgebend für die Verteilung von Ressourcen

I Der Beitrag wurde im Rahmen des Forschungsprojekts „Ein Staatssekretariat für Integration: Integrationspolitik in Bewegung?“ am Institut für Politikwissenschaft der Universität Wien erarbeitet, unterstützt durch FördergelderdesJubiläumsfonds derÖsterreichischen Nationalbank (Projektnummer: 15758). Besonderer Dank gilt der Projektleiterin Prof. Sieglinde Rosenberger für ihre Kommentare zum vorliegenden Beitrag, Veronika Heider für ihre Mitarbeit an der Erhebung sowie den GutachterInnen für ihre Anmerkungen. und sozialen Statuspositionen betrachtet werden (Rieger 2005). Dieser Anspruch steht in engem $\mathrm{Zu}-$ sammenhang mit Gerechtigkeitsansprüchen an gesellschaftliche Ordnung - Leistung grundsätzlich zu belohnen und nicht zu ignorieren gilt als in vielen Kulturen anerkanntes Motiv (Sen 1999). Leistung ist allerdings kein selbsterklärender Begriff, vielmehr bedarf es jeweils thematischer Spezifikationen dessen, was als Leistung zu verstehen ist, was anerkannt und honoriert wird. In unterschiedlichen historischen Konstellationen kam seine Bedeutung dabei in je eigener Form zum Ausdruck, etwa in den Forderungen des frühen Bürgertums gegenüber den Privilegien des Adels, als liberalistisches Verteilungsprinzip moderner kapitalistischer Wirtschaftsordnung, oder in den Ansprüchen der ArbeiterInnen- und Frauenbewegungen, das Spektrum anzuerkennender Leistungen um industrielle Lohnarbeit sowie Reproduktions- und Hausarbeitsleistungen zu erweitern (Degele/Winker 20II, 37). Aufgrund dieser Variabilität übernehmen Leistungskriterien daher eine wesentliche Funktion für die Verfestigung hegemonialer Interessen derer, welche über sie bestimmen können (Young 1990, 193).

Meritokratische Ansätze üben zudem eine zentrale Funktion in der Konstitution von Gruppenzugehörigkeiten und -hierarchien aus. Anstelle von askriptiven Merkmalen wie Geschlecht, Ethnizität, Klassenhintergrund, etc. kategorisiert es Menschen entlang erarbeiteter Eigenschaften („Leistungen“) und ist damit entscheidend verantwortlich für deren In- und Exklusion in/von bestimmte/n Statusgruppen. Dass askriptive Merkmale aber einen hohen Einfluss auf individuelle Leistungsmöglichkeiten haben und dass Leistungsgerechtigkeit daher auch Chancengleichheit in den Ausgangsbedingungen von Menschen verlangt, war ein zentrales Element der Meritokratiekritiken der I970er-Jahre (z.B. Rawls 1972). Dieser Gesichtspunkt ist insbesondere dort von zentraler Bedeutung, wo strukturell benachteiligte und von Diskriminierung betroffene Gruppen mit dem Anspruch eines Leistungsprinzips konfrontiert werden, so eben auch MigrantInnen im Kontext von Integration.

In Politikfeldern wie der Arbeitsmarkt-, Sozialoder Bildungspolitik prägt das Leistungsprinzip die facheinschlägige Debatte seit längerem und wird in den letzten Jahrzehnten angesichts veränderter Arbeits- und Kapitalmarktbedingungen (Privatisierung, Deregulierung, Globalisierung) (Bischoff 2005), im Lichte der Transformation von Wohlfahrtsstaatlichkeit zwischen fördernder und fordernder Tendenz (Lahusen/Stark 2003) sowie im Rahmen wiederkehrender Bildungsreformdebatten (Opielka 2005) zu einem immer gewichtigeren Referenzpunkt (siehe dafür auch die weiteren Beiträge dieses Themenhef- 
tes). In der integrationspolitischen Forschung wurde das Leistungsprinzip bislang hingegen kaum systematisch behandelt oder gar hinsichtlich seiner inklusiven wie exklusiven Folgen beforscht (erste Auseinandersetzungen damit finden sich z.B. bei Castilla 2008; Jørgensen/ Thomsen 2013; Lanz 2009; Moore 2000; Segert 2008). Gerade in sich neu entwickelnden Politikfeldern wie jenem der Integration werden aber noch stärker als in tradierten Feldern politische Kämpfe um die Definition von Leistungsverständnissen geführt, da diese hier noch formbarer sind (Massey/Huitema 2012). Dies macht sie für die Analyse der praktischen Anwendung des theoretischen Leistungsprinzips besonders geeignet: In Frage steht dabei, welche Kriterien als legitime Leistung anerkannt und inwieweit diese aus anderen Politikfeldern abgeleitet werden. Darüber hinaus ist für die Betrachtung meritokratischer Politiken generell bedeutsam, ob und inwiefern definierte Leistungen auch zum Einund Ausschlusskriterium für die Teilhabe an welchen sozialen Systemen gemacht werden (Kronauer 20IO), denn nicht zuletzt darin unterscheiden sich belohnende von bestrafenden Ansätzen in der Gesellschaftspolitik (Foucault 1994; French/Raven 1959; Oliver 1980).

Schließlich ist aus politikwissenschaftlicher Sicht zu klären, ob Leistung lediglich implizites Merkmal steuerungspolitischer Maßnahmen ist oder als explizites, sinngebendes Leitmotiv politischer Gestaltung verstanden wird. Während kaum ein politisches Gestaltungskonzept völlig ohne einen, zumindest impliziten, Anspruch an Leistungsgerechtigkeit auszukommen vermag, steht ein explizit meritokratischer Politikansatz vor der Herausforderung, seine Leistungskriterien und deren Legitimitätsanspruch kenntlich machen und plausibel begründen zu können.

\subsection{Integrationspolitik in Österreich: Ein Politikfeld im Mehrebenensystem}

Die politische Steuerung der Integration von MigrantInnen war lange kein dominantes politisches Thema. Während GastarbeiterInnen-Programme quer durch Europa bereits in den 196oer-Jahren Zuwanderung regelten, hinkten integrationspolitische Begleitmaßnahmen nicht zuletzt aufgrund der auf Rückkehr ausgelegten Migrationskonzeption - lange Zeit hinterher (Bauböck 1996; Mourão Permoser/Rosenberger 2012; Perchinig 20IO). In Österreich hatten sich zuerst die Kommunen und Länder der Integration angenommen und entwickelten bereits früh institutionalisierte Formen von Integrationspolitik, bundespolitisch wurde kein Bedarf nach systematischer Integrationspolitik gesehen, da man sich nicht als Einwanderungsland verstehen wollte (Perchinig 20IO; Kraler 20II). Erst im Laufe der 1990erJahre führten die steigende Zahl an Nicht-StaatsbürgerInnen infolge von Arbeitsmigration, Familiennachzug und Flucht/Asyl, sowie die zunehmende Politisierung des Themas durch die Oppositionsparteien FPÖ und Grüne zu einer bundesweiten Diskussion über (fehlende) Integration von MigrantInnen in Österreich (Gruber 20I4; Mourão Permoser/Rosenberger 2012; Strasser/ Tošić 20I3). Integration wurde zum negativ konnotierten, politischen Reizbegriff, der im Wesentlichen der Legitimierung restriktiverer Zuwanderungs- und Asylgesetze diente (Krzyżanowski/Wodak 2008). Zusammen mit einem restriktiven Staatsbürgerschaftsregime, in dem Bund und Länder geteilte Kompetenzen aufweisen (Stern/Valchars 20I3), führte dies zu einer schwierigen integrationspolitischen Ausgangslage. Dominierend war zunächst ein assimilatorisches Integrationsverständnis, das auf die kulturelle Dimension von Integration abzielte, d.h. auf die Anpassung an Sprache, Werte und Normen der einheimischen Bevölkerung (Volf/Bauböck 200I). Mit den von der Großen Koalition 1997 als „Integrationspaket“ betitelten Beschlüssen (BGBl. I Nr. 75-78/1997) versuchte man das Leitprinzip „Integration vor Neuzuzug " in Zuwanderungs-, Niederlassungs- und Asylgesetzen zu verankern. Dazu wurde die Aufenthaltssicherheit für Drittstaatsangehörige gestärkt, während beschäftigungs- bzw. sozialrechtliche Seiten der Integration nur in geringem Ausmaß angesprochen und andere Bereiche gänzlich ausklammert wurden (König 1998, 154) - Integration blieb also mehr Etikett denn Inhalt (Pelinka/Rosenberger 2007, 42).

Mit der seitens der ÖVP/FPÖ-Regierung als symbolisches Signal eingeführten „Integrationsvereinbarung“ im Jahr 2003 wurde der Erwerb von Deutschkompetenzen (A2) gegenüber Neuzuwanderern als Voraussetzung für längerfristigen Aufenthalt festgeschrieben sowie das Angebot an Sprachkursen sowie - prüfungen bundesweit vereinheitlicht und der Verantwortung des aufgewerteten „Österreichischen Integrationsfonds“ (ÖIF) übertragen (Götzelmann 20IO; Mourão Permoser 2012). Diese Maßnahmen lagen in einem europäischen Trend der Einführung von "civic integration“ Programmen, welche die für Einbürgerung benötigte Kriterien nun sukzessive auch auf andere Gruppen von MigrantInnen wie Daueraufenthaltsberechtigten bzw. auf Neuzuwanderung generell ausweiteten (Goodman 20I4; Joppke 2007; Michalowski/van Oers 20I2; Mouritsen 20I3). Bereits hier fanden sich also Ansätze für ein Kriterien-geleitetes Modell der Integrationspolitik, aufbauend auf spezifischen $\mathrm{zu}$ erbringenden Leistungen. Allerdings wurden diese (noch) nicht explizit als Teil eines meritokratischen Integrationsmodells kommuniziert sondern sollten einer unterstellten „Integrationsunwilligkeit“ entgegenwirken (Mourão Permoser 2012) und gleichzeitig migrationspolitisch selektierend wirken (Perchinig 2012, 36).

Unter der Wiederauflage der Großen Koalition zwischen SPÖ und ÖVP wurden Schritte zu einem komplexeren integrationspolitischen Ansatz gesetzt, erneut im 
Lichte von Impulsen auf Europäischer Ebene (u.a. der Einigung auf "Common Principles" der Integration von Drittstaatsangehörigen sowie der Einführung eines Europäischen Integrationsfonds): Hier sind v.a. die Initiierung einer "Integrationsplattform“ zum Austausch von ExpertInnen und Stakeholdern aus der österreichischen Integrationslandschaft (2008), die Einrichtung eines Integrationsbeirats sowie ExpertInnenrats (2010) und die Entwicklung eines „Nationalen Aktionsplans für Integration" (NAP.I) (20I0) zu nennen, in welchem relevante Handlungsfelder der Integrationspolitik identifiziert wurden, von Sprache und Bildung über Arbeit, Soziales und Gesundheit bis hin zu Rechtsstaat, Werten und interkulturellem Dialog.

Mit der Einrichtung eines Staatssekretariats für Integration (SSI) wurde ein meritokratischer Ansatz schließlich zum expliziten Leitgesichtspunkt der Integrationspolitik auf Regierungsebene. 20II im Zuge einer Regierungsumbildung etabliert, blieb das SSI formal dem - von der ÖVP geführten - Bundesministerium für Inneres (BMI) untergeordnet, in dem bis dahin wesentliche integrationspolitische Kompetenzen angesiedelt waren. Erstmals war nun ein eigenes Regierungsmitglied spezifisch für Integration als Politikfeld verantwortlich. Aus parteipolitischer Perspektive baute die ÖVP mit diesem Schritt ihr regierungsinternes "Ownership“ im Themenbereich Integration gegenüber dem Koalitionspartner weiter aus, was nicht nur die Bemühungen der SPÖ um eigene integrationspolitische Linie ausbremste. ${ }^{2}$ Es sorgte in der Folge auch für Spannungspotenzial zwischen SPÖ-geführten Ministerien und dem Staatssekretariat, die in der Querschnittsmaterie Integration nun auf noch engere Zusammenarbeit angewiesen waren. Trotz fortbestehender Ressortverantwortlichkeit der Fachministerien bemühte sich der neue Akteur um einen neuen "Blick“ auf Integration unter dem von nun an propagierten Ansatz „Integration durch Leistung“ dieser steht im Zentrum der folgenden Analyse.

2 Im Rahmen ihrer Programmdiskussion "Österreich 2020" hatte sich die Bundes-SPÖ die Erarbeitung eines eigenen Integrationskonzeptes auf die Agenda gesetzt. Auf Basis eines Präsidiumsbeschlusses („Zusammenleben nach klaren Regeln“) war eine Arbeitsgruppe rund um Bundeminister Norbert Darabos mit der Entwicklung bis zum SPÖ-Parteitag 2010 beauftragt worden, ein entsprechendes konkretes Konzept lag jedoch auch zum Zeitpunkt der Einrichtung des SSI noch nicht vor. (Der Standard, 23.09.2009, S.6; Der Standard, I4.I0.2010, S.7; Die Presse, 25.06.2011, S.8). Pläne für ein eigenes Integrationsstaatssekretariat (etwa im Bundeskanzleramt) waren seitens der SPÖ zwar wiederholt geäußert worden, trafen jedoch auf deutlichen Widerstand seitens der für das BMI zuständigen ÖVP (Die Presse, I2.IO.20IO, S.4).

\section{Material und Methoden}

Die vorliegenden Forschungsergebnisse stammen aus einem vom Jubiläumsfonds der Österreichischen Nationalbank geförderten Forschungsprojekt am Institut für Politikwissenschaft der Universität Wien. Das Projekt untersucht den Einfluss des 201 in eingerichteten Staatssekretariats für Integration auf die Gestaltung der Österreichischen Integrationspolitik.

Die hier präsentierte Untersuchung des meritokratischen Gestaltungsprinzips der Integrationspolitik basiert auf zwei Analyseebenen: Erstens wird die diskursive Ebene politischen Handelns, in Form der politischen Erzählung (Narrativ) "Integration durch Leistung“, nachgezeichnet. Zweitens werden politische Maßnahmen (Policies) untersucht. Diese verdeutlichen, wie abstrakte Leistungsvorstellungen durch spezifische Leistungskriterien konkretisiert werden. Dabei berücksichtigen wir sowohl in Planung befindliche als auch bereits beschlossene Maßnahmen, weisen aber auf den jeweiligen Umsetzungsstand hin (siehe Tabelle I). Dies erlaubt in einem dritten Schritt, Konsistenzen und Brüche des meritokratischen Ansatzes zwischen integrationspolitischem Diskurs und Maßnahmen aufzuzeigen.

In einem vergleichenden Design wurden in der Studie zwei Phasen der 25. Legislaturperiode des österreichischen Parlaments (2008-20I3) gegenübergestellt, die Phase vor (2008-20II) sowie die Phase nach (2OII-2OI3) Einrichtung des SSI. Da das Forschungsinteresse den Veränderungen der Regierungspolitik infolge der institutionellen Neuerung galt, wurden weder Daten über den öffentlichen Diskurs im Allgemeinen noch über Reaktionen anderer politischer Akteure außerhalb der Bundesregierung erhoben. Die diesem Beitrag zugrundeliegende empirische Basis setzt sich aus Medienberichten (Die Presse, Der Standard), Presseaussendungen (des SSI bzw. der Bundesministerien für Inneres, Arbeitund Soziales sowie Unterricht, Kunst und Kultur), ministeriellen Integrationsberichten sowie integrationsbezogenen Plenardebatten im Nationalrat und Gesetzesbeschlüssen des Nationalrats zusammen. Materialen aus der medialen und parlamentarischen Arena wurden auf Basis von Narrativanalyse (Shanahan et al. 20II) und Claims-Analyse (Koopmans 2002) untersucht, für Gesetzesbeschlüsse und Policies wurde eine Dokumentenanalyse nach analogen Gesichtspunkten durchgeführt. Unter Policy-Narrativen verstehen wir mit Kaplan (1986) diskursiv vermittelte politische Erzählungen, in denen komplexe Situationen durch die Bereitstellung grundlegender Erzählelemente gerahmt und mit Lösungsstrategien verknüpft werden. Als Claims werden strategische Forderungen politischer AkteurInnen im öffentlichen Raum verstanden, durch welche eine politische Meinung artikuliert wird (Koopmans 2002), im konkreten Fall politische Forderungen des SSI. Die qualitative Codierung 
der Materialien berücksichtigte dabei u.a. jeweils Adressaten, Inhalt und Ausrichtung von Forderungen/Erzählelementen/Gesetzesbeschlüssen, Integrationsdimensionen und Formen der Politikgestaltung. Die Befunde der Materialanalysen wurden durch ExpertInneninterviews mit führenden BeamtInnen aus SSI, BMI sowie dem österreichischen Integrationsfonds validiert und ergänzt.

\section{4. „Integration durch Leistung“. Empirische Befunde zu Diskurs und Policy}

\section{1 „Leistung“ als integrationspolitische Erzählung}

Policy-Narrative erfüllen die zentrale Funktion, komplexe Themen in einen leicht nachvollziehbaren Bedeutungszusammenhang zu setzen, um einfache Lösungen für vielschichtig Probleme anbieten zu können. Nach Kaplan (1986, 770) vermitteln sie "who should do what, and how, when and why they should do it in order to address policy dilemmas". Wenngleich sie nicht unbedingt mit einer Bezugnahme auf Fakten einhergehen müssen, ist für politische Narrative eine innere Konsistenz bei gleichzeitiger Flexibilität in der Interpretation verschiedener Problemlagen essentiell (Boswell et al. 20II). Die politische Narrativanalyse untersucht den Aufbau solcher Erzählungen, um die diskursive Konstruktion von Politikproblemen und Lösungsansätzen nachvollziehen zu können.

Ausgehend von der Annahme, dass Narrativ-Strukturen in Anlehnung an literarische Erzählungen analytisch erfasst werden können, wird nach Shanahan et. al (20II) im Narrativ zwischen Plot, Charakteren, Lösungen und kausalen Mechanismen unterschieden. Der Plot eines Narrativs erfasst das Policy Problem, verbindet die einzelnen Erzählelemente und setzt sie in einen Verstehenskontext. Die Erzählung beinhaltet Charaktere, also die handelnden Personengruppen, Handlungsanweisungen und Lösungsansätze, die mittels behaupteter Kausalzusammenhänge in Beziehung gesetzt werden. Im Folgenden wird dieser Ansatz auf das Narrativ der österreichischen Integrationspolitik angewandt.

Plot

Ausgangspunkt für die politische Erzählung „Integration durch Leistung " ist der vom Staatssekretariat gestellte Problembefund eines Politikversagens im Bereich Integration. Das neue Narrativ grenzt sich von bisherigen, auf ethnische und kulturelle Unterschiede fokussierten Integrationszugängen ab. Betont wird vom neuen Akteur, dass Integrationspolitiken bisher zu stark ideologisch geprägt gewesen seien und daher ein entpolitisierter Zugang, vom SSI als "Versachlichung" bezeichnet, notwendig wäre (Der Standard 2012; Die Presse 2013).
Ein solcher sachlicher Zugang sei in einem Fokus auf Leistung zu finden:

„Es soll nicht die Herkunft eines Menschen zählen, sondern die Leistung, die jemand erbringt. Integration geschieht letztendlich durch Leistung - egal ob im Beruf, im Verein oder in der Schule."(Staatssekretariat für Integration 2OII).

Leistungsforderungen finden sich zwar bereits in früheren Wortmeldungen des Innenministeriums (OTS 2OIO), als umfassende politische Erzählung besitzt das Narrativ "Integration durch Leistung" jedoch wesentlichen Neuerungswert.

Das oben angeführte Zitat aus dem Leitbild des SSI verdeutlicht, dass von MigrantInnen erbrachte Leistung in verschiedenen Bereichen nun als direkter Weg zur Integration konstruiert wird. Die politische Erzählung ist aber umfassender und betont die Notwendigkeit von Forderung, Förderung und Anerkennung bzw. Wertschätzung erbrachter Leistung. Der Logik des Narrativs folgend, resultieren Integrationsdefizite daher nicht nur aus von MigrantInnen nicht erbrachten Leistungen sondern auch aus mangelhaft erfolgter Ermöglichung, Anerkennung und Einforderung ebensolcher.

\section{Charaktere}

Im Narrativ "Integration durch Leistung" findet sich eine umfassende Ansprache verschiedener HandlungsträgerInnen. Dazu gehören sowohl MigrantInnen und Menschen mit "Migrationshintergrund“ einerseits als auch Mehrheitsgesellschaft sowie Politik andererseits. Wenngleich ihnen als AkteurInnengruppen im Leistungsnarrativ unterschiedliche Rollen zugeschrieben werden, beruht das Verständnis von Integration zunächst auf Beidseitigkeit, also auf einem von allen Mitgliedern der Gesellschaft getragenen Prozess:

„Wir können an Menschen mit Migrationshintergrund den Anspruch stellen, dass sie in Österreich leistungswillig sind und diese können an uns den Anspruch haben, dass wir ihre Leistung annehmen." (OTS 2OII).

Im Zentrum der Erzählung stehen Menschen mit Migrationsgeschichte, als Subjekt wie Objekt von Integrationsleistungen. Ihnen wird sowohl die Aufgabe zugewiesen, politische Integrationsmaßnahmen wahrzunehmen als auch darüber hinaus eigeninitiativ Leistung zu erbringen. Dabei wird gezielt auf Ebene des Individuums angesetzt d.h. an der individuellen Leistung, Leistungsfähigkeit und Leistungswilligkeit von MigrantInnen. Ein besonderer Fokus liegt dabei auf Kindern und Jugendlichen: 
„Auch an Schulen muss gelten: Nicht die Herkunft, sondern die Leistungsbereitschaft und das eigene Engagement der Kinder und Jugendlichen zählt." (OTS 2012b)

Die Mehrheitsgesellschaft wird als AkteurInnengruppe in der Regel als ein nicht näher definiertes „wir" adressiert, politische AkteurInnen dagegen häufig spezifischer angesprochen. So wird ihnen nicht nur das zu Beginn angesprochene „Politikversagen“ zugeschrieben, sondern auch die Verantwortung für die Herstellung von „leistungsbefördernden“ Bedingungen:

„Natürlich muss Leistung auch möglich gemacht werden, das heißt, die Politik muss Rahmenbedingungen schaffen."(OTS 20I2a).

In der Analyse der Charaktere der politischen Erzählung „Integration durch Leistung" zeigt sich, dass Integration als multipler Anpassungsprozess verstanden wird. Die zu erbringenden Leistungen sind zwar ungleich verteilt, auf der narrativen Ebene findet man dennoch eine Ansprache aller Charaktere der Erzählung und eine Betonung der Beteiligung aller AkteurInnengruppen um Integrationsziele zu erreichen.

\section{Lösungsansätze}

Die im Narrativ transportierten Lösungen sprechen Integrationsprobleme auf der Makro-Ebene an und enthalten somit grundsätzliche Überlegungen, wie Integrationsprobleme gelöst werden sollen. Es lassen sich verschiedene Bereiche identifizieren, in denen die unterschiedlichen Charaktere der Erzählung Leistungen als Lösung einbringen sollen, wobei das Narrativ einen klaren Schwerpunkt auf den Bereichen Sprache/Bildung, Erwerbsarbeit und Ehrenamt zeigt.

Die mit Bildungsleistungen verknüpfte Erwartung ist nicht nur jene einer leichteren Sozialintegration durch bessere Sprachfähigkeiten, sondern auch eines positiven Zusammenhangs mit der Leistungsfähigkeit im Beruf. Als Begründung für Bildungsleistungen findet sich auch die Vermeidung sozialstaatlicher Folgekosten:

„,Wir investieren in Österreich derzeit sehr viel in das spätere Reparieren. Es wird Zeit, dass wir anfangen, in das frühere Fördern zu investieren. Vor allem in die sprachliche Frühförderung, sagt Kurz."(OTS 2OI2c).

Neben Bildung werden insbesondere Arbeitsleistungen als wesentlicher Beitrag zur Integration skizziert, einerseits aufgrund ihres Identität und Gemeinschaft stiftenden Charakters, vor allem aber im Sinne der Gewährleistung der Selbsterhaltung. Ehrenamt wird als Indikator sozialer Integration von MigrantInnen in ihrem Lebensumfeld verstanden, die zudem einen anerkennungswürdigen Mehrwert für das Gemeinwohl erzeugt.

\section{Kausalzusammenhänge}

Die argumentative Verbindung von Problemen und Lösungen mittels kausaler Mechanismen ist ein Charakteristikum (erfolgreicher) politischer Erzählungen. Im Narrativ „Integration durch Leistung“ werden Lern- und Bildungsleistungen, ökonomische Leistungen sowie ehrenamtliche Leistungen als Lösung für ökonomische Benachteiligungen konstruiert, die Anerkennung, Einforderung und Ermöglichung dieser Leistung als Weg dahin. Die kausalen Mechanismen des Narrativs reichen aber darüber hinaus: Mit dem Argument, dass Leistungen in den Bereichen Sprache/Bildung, Erwerbsarbeit und Ehrenamt auch zu sozialer Anerkennung und damit zu Zugehörigkeitsgefühlen führt, wird schließlich auch identifikatorische Integration in einen Leistungskontext gesetzt:

„Sebastian Kurz: Wer Anerkennung will, muss sie sich durch Leistung erarbeiten. Jene, die Deutsch lernen, eine gute Ausbildung machen, etwas leisten und sich am gesellschaftlichen Leben beteiligen, sind angekommen." (Die Presse 20IIa)

Solche Kausalketten suggerieren, dass der Ansatz in der Lage ist, grundsätzlich jedes Problem im Politikfeld zu adressieren und auch zu lösen. Sie erfüllen damit eine wesentliche Voraussetzung für die Durchsetzungsfähigkeit politischer Erzählungen (Shanahan et al. 20II, 540).

\section{Erzählstränge außerhalb des Narrativs "Integration} durch Leistung“

Obwohl identifikatorische Integration als Konsequenz von „Leistung" konstruiert wird, lässt sich im Diskurs auch ein auf Werte und Normen bezogener Erzählstrang, abseits des Narrativs „Integration durch Leistung" identifizieren. Erverlangt nach Anpassung seitens MigrantInnen, bezogen auf Kultur, Rechtsordnung und kollektive Werte. Identifikatorische Integration wird somit innerhalb des Leistungsnarrativs (als erwartbare Folge im Kausalzusammenhang mit erbrachter Leistung), aber auch separat und ohne Ansprache von Leistung adressiert. Der narrative Anspruch einer Abkehr von der bisherigen kulturalistischen Integrationspolitik wird somit nur zum Teil realisiert. Dies bestätigt sich, wenn man die konkreten integrationspolitischen Maßnahmen seit dem diskursiven Fokus auf Leistungspolitik analysiert.

4.2 Die Konkretisierung der Erzählung: Integrationsmaßnahmen unter dem Leistungsgesichtspunkt

Die Umsetzung politischer Narrative in Maßnahmen kann unterschiedliche Formen annehmen, wie z.B. gesetzliche Regelungen, die Förderung von Projekten, die Lancierung von Kampagnen, etc. (Bemelmans-Videc et al. 20I0). In diesem Beitrag geht es um die Frage, mit 
Tabelle 1: Integrationspolitische Maßnahmen nach Leistungsdimension, 2011-2013

Lern-/Bildungsleistungen

Spracherwerb:

- "Crash-Kurse“ für Schul-Quereinsteiger mit Defiziten

- Fortführung begleitender Sprachförderkurse in allen Schulstufen

- Förderung außerschulischer Lernprojekte wie Lerncafés der Caritas

- Assistenzpakete für Deutsch vor Zuzug (Infomaterialien über Leistungsvoraussetzungen, Sprachinstitute und Lehrmaterialien)

- Deutschkurs-Initiativen für Erwachsene

- Berufsspezifische Deutschkurse für Pflegekräfte

- Verschärfung der Integrationsvereinbarungskriterien: Nachweis eines höheren Sprachniveaus (A2) als bisher (A1) nun bereits binnen 2 Jahren;

- Verpflichtende Vorschulklassen bei Deutschdefiziten (t.r.)

- Zweites verpflichtendes Kindergartenjahr (n.r.)

- Deutsch-Kenntnisse als Voraussetzung für gesetzliche Schulreife (n.r.)

- Vorzeitige Staatsbürgerschaft für „ausgezeichnet Integrierte“ (Fast-Track)

Sonstige Schul- und Erwachsenenbildung:

- Paket gegen Schulpflichtverletzung (u.a. Einbeziehung von Sozialarbeitern; gesetzliche Fixierung elterlicher Verantwortung; Verwaltungsstrafen)

- Kampagne „Zusammen Österreich - Deine Chance!

- Gesetzliche Erleichterung der Nostrifizierung akademischer Abschlüsse

- Einrichtung von Nostrifikations-Anlaufstellen

- Förderung von Erwachsenenlehrgängen (Erlangung von Basiswissen; Nachholung des Pflichtschulabschlusses)

- Bildungspass für Niedergelassene

- $\quad$ Ausweitung der Schulpflicht zur Bildungspflicht bis zum 18. Lebensjahr (n.r.)

\section{Erwerbsarbeitsleistung}

- Punktebasiertes Systems der Arbeitsmigration (Rot-WeißRot-Karten) und Welcome-Desks für Neuzuwanderer

- MigrantInnen-Index des Arbeitsmarktservice (AMS)

- Jugend-/Lehrlingscoaching

- Berufsspezifische Förderprogramme (z.B. ,Biber'-Akademie, ,DiePresse'-Migrant/innenlehrgang, ,MigrantsCare'-Projekt)

- „Mentoring“-Programm für Jungunternehmer

- „Zusammen Österreich - IntegrationsbotschafterInnen“

- Vorzeitige Staatsbürgerschaft für „ausgezeichnet Integrierte“ (Fast-Track)

\section{Ehrenamtliche Leistungen}

- Gesetzliche Öffnung Freiwilliger Feuerwehren für MigrantInnen

- „Zusammen Österreich - Jetzt du! Dein Land braucht dich!“

- Integrationspreis Sport

- Fördertopf für Integration im Rahmen der BundesSportförderung

- Projektförderung „MiMi-Gesundheitslotsinnen“

- Vorzeitige Staatsbürgerschaft für „ausgezeichnet Integrierte" (Fast-Track)

Identifikatorische Integration

- „Rot-Weiß-Rot-Fibel“ als normativer Orientierungsrahmen für MigrantInnen

- Reform des Staatsbürgerschaftstests (Fokus auf verfassungsmäßige Grundprinzipien für Gesetzgebung und gesellschaftliches Zusammenleben in Österreich)

- Festlichere Gestaltung des Verleihungsaktes

- Forderung nach „Deutsch in Moscheen“ (n.r.)

- Gesetzliche Verschärfung der Strafverfolgung von

Zwangsehe \& Genitalverstümmelung

- Einrichtung eines „Dialogforum Islam“

- Novellierung des Islamgesetzes

\section{Sonstige Integrationsbereiche}

- Verankerung eines Integrationskoordinators im Hauptverband der Österreichischen Sozialversicherungsträger

- Auszeichnungen für interkulturelle/integrative Vermittlungsleistungen (z.B. ,Journalistenpreis Integration', ,Integrationspreis Sport')

- Migrationssensible Hausverwaltung (t.r.)

- Desegregierendes Wohnungsmanagement (n.r.)

Anmerkungen: Die Analysegrundlage bilden integrationsrelevante Gesetzesbeschlüsse des Nationalrats sowie Integrationsprojekte und -kampagnen, soweit sie im Diskurs der untersuchten Ministerien - in Medienartikeln, Presseaussendungen sowie ministeriellen Informationsmaterialien (z.B. Integrationsberichte) - wiederholt angesprochen und damit als für die eigene Arbeit wesentlich qualifiziert wurden. Nicht realisierte (n.r.) bzw. nur teilweise realisierte (t.r.) Maßnahmen sind entsprechend gekennzeichnet. 
welchen konkreten Forderungen und Maßnahmen der narrative Leistungsanspruch umgesetzt wird bzw. welches Verständnis von "Integrationsleistungen“ darin zum Ausdruck kommt. Sowohl die Claims-Analyse der gestellten Forderungen als auch die Untersuchung der Maßnahmen in Gesetzesbeschlüssen zeigen, dass auch hier Bildungs- und Arbeitsleistungen als primäre Integrationsvehikel dominieren. Tabelle I zeigt die gesetzten und geforderten Maßnahmen, die den im Narrativ identifizierten Arten von Leistung zugeordnet wurden, ebenso wie außerhalb des Leistungsnarrativs kommunizierte Politikforderungen/-maßnahmen.

\subsubsection{Lern-/ Bildungsleistungen}

Der an alle Altersgruppen gestellte Leistungsanspruch besteht in Maßnahmen für Bildung und Spracherwerb. Bereits in der frühkindlichen Bildungsentwicklung sollen Sprach- und Lernkompetenzen gefördert werden, weshalb vom SSI eine Ausweitung der bereits 2010 eingeführten einjährigen Verpflichtung für alle Kinder auf ein zweites verpflichtendes Kindergartenjahr gefordert wird, allerdings nur für Kinder mit Sprachdefiziten (bis dato lediglich in Modellregionen erprobt). Ähnliche Ansprüche kommen in der Forderung nach verpflichtenden Vorschulklassen für Kinder mit Deutschdefiziten zum Ausdruck, auch sie wurden aufgrund koalitionären Konflikts nur als abgeschwächte Kompromissvariante (Schulstandortvariabel) umgesetzt. Im Pflichtschulalter werden verschiedene Sprachförderungsmaßnahmen angeboten, die auf die Anforderungen des allgemeinen schulischen Leistungsniveaus vorbereiten sollen („Crash-Kurse“ für Quereinsteiger in Volksschulen, Weiterführung begleitender Sprachförderkurse in allen Schulstufen oder außerschulische Lernprojekte wie die Lerncafés der Caritas). Deutsch-Kenntnisse werden vom SSI sogar als Voraussetzung für die gesetzliche Schulreife angedacht, allerdings nicht ernsthaft weiterverfolgt. (Aus-)Bildung wird aber auch über den Spracherwerb hinaus als Leistungsgut bewertet, etwa im Rahmen eines Maßnahmenpakets gegen Schulpflichtverletzungen, das die Bildungsintegration von Kindern mit Migrationshintergrund zum expliziten Ziel hat. Mit Kampagnen wie „Zusammen Österreich - Deine Chance!" sollen SchülerInnen mit Migrationsgeschichte nicht zuletzt durch so genannte "Integrationsbotschafter" positive Vorbilder finden.

Fördermaßnahmen in der Erwachsenenbildung werden vom SSI primär dort angesprochen, wo Erwachsene bestimmte Grundfähigkeiten noch nicht erworben haben. Dem soll durch Erwachsenenlehrgänge zur Nachholung von Basiswissen bzw. des Pflichtschulabschlusses als auch durch Deutschkurs-Initiativen oder einen „Bildungspass“ für MigrantInnen mit gesichertem Aufenthaltsstatus entgegengewirkt werden. Auch im Erwachsenenbereich gilt die Sprachleistung als wesent- liche Integrationsdimension: So sind künftig bereits vor Zuzug grundlegende Deutschkenntnisse (AI) zu erbringen, dafür werden eigene „Assistenzpakete“ (Informationsmaterialien) aufgelegt. Nach der Einreise stehen Sprachleistungen nicht zuletzt im Zentrum der 2OII verschärften Integrationsvereinbarung, die von Neuzuwandernden nun binnen zwei Jahren den Nachweis eines höheren Sprachniveaus (A2) als bisher (AI) festsetzt. Bereits im Ausland erbrachte (Aus-)Bildungsleistungen versucht man durch die gesetzlich erleichterte Nostrifikation von Zeugnissen und Bildungsabschlüssen sowie durch die Einrichtung von Informationsstellen zu nutzen. Bislang wurde dies aber lediglich für akademische Bildungsabschlüsse realisiert, eine Ausweitung auf andere, nicht-akademische Ausbildungstitel befindet sich noch in Diskussion. Damit ist die zweite zentrale Leistungsdimension kapitalistischer Gesellschaften angesprochen: ökonomische Leistung im Erwerbssystem.

\subsubsection{Erwerbsarbeitsleistung}

Im Narrativ, wie bei den konkreten Maßnahmen steht der Arbeits- und Selbsterhaltungsaspekt im Zentrum. Das Schwergewicht der Maßnahmen liegt dabei auf klassischer Erwerbsarbeit. Die Einführung des punktebasierten Systems der Arbeitsmigration (Rot-Weiß-RotKarten) steht in der Logik einer auf Leistungsfähigkeit im Arbeitsmarkt ausgerichteten Zuwanderung, welche die spätere Integrationsfähigkeit begünstigen soll. Dies korrespondiert mit einer seit den 1980er-Jahren beobachtbaren Reorganisation des Wohlfahrtsstaatsmodelles der Nachkriegszeit, in der universelle Integrationsansprüche des Staates gegenüber seinen BürgerInnen in Richtung individualisierter und auf persönlichen Vorleistungen (insb. Steuerabgaben) angepasster Integrationsangebote verändert werden (Bommes 2004, 158). Gegenüber MigrantInnen wird die Gewährleistung sozialstaatlichen Schutzes als "club good“ durch sich immer stärker ausdifferenzierende Zuwanderungsund Aufenthaltstypen reglementiert (Kolb 2008, I25f.). Folglich zählen zu den mit den Rot-Weiß-Rot-Karten für MigrantInnen eingeführten Leistungskriterien neben allgemeinen Voraussetzungen wie Sprachkompetenzen und Selbsterhaltungsfähigkeit (Einkommen) auch spezifischere, durch eigene Aufenthaltstitel abgedeckte Kriterien wie nachgefragte Berufskompetenzen in Mängelberufen oder überdurchschnittliche Fertigkeiten von Hochqualifizierten. Neu eingerichtete Welcome-Desks versorgen NeuzuwandererInnen mit entsprechenden Informationen.

Für die berufliche Integration bereits ansässiger MigrantInnen werden Maßnahmen in den arbeitsmarktund wirtschaftspolitischen Förderungssystemen gesetzt, so etwa ein vom österreichischen Arbeitsmarktservice (AMS) eingeführter MigrantInnen-Index zur gezielten Identifizierung migrantischer ArbeitnehmerInnen - 
eine Reaktion auf die seitens der EU verlangten Zielwerte für MigrantInnen für die Vergabe von Mitteln des Europäischen Sozialfonds. Ein Jugend-/Lehrlingscoaching wird vom SSI als berufsbezogene Integrationsmaßnahme hervorgehoben, ebenso wie neu geschaffene berufsspezifische Förderprogramme für MigrantInnen (etwa die ,Biber'-Journalismusakademie, der ,DiePresse'-Migrant/innenlehrgang oder das ,MigrantsCare'-Projekt). Schließlich wird auch der Sektor Selbständige und KMU adressiert, etwa durch das „Mentoring“-Programm für Jungunternehmer.

Nicht zuletzt wird auch in der Staatsbürgerschaftspolitik ein Modell der "Staatsbürgerschaft durch Leistung" präsentiert (SSI in Der Standard, 2OI3), das stark auf Erwerbsarbeitsleistungen Bezug nimmt. Damit wird ein Ansatz beibehalten, der Staatsbürgerschaft nicht als Mittel zur Integration, sondern als Endpunkt eines bereits erfolgten Integrationsprozesses versteht. Bereits vor der Einrichtung des SSI war der Zugang zur Staatsbürgerschaft wie auch $\mathrm{zu}$ vielen unbefristeten Aufenthaltstiteln jenen vorbehalten, die ein gesichertes Einkommen ohne Inanspruchnahme bedarfsgeprüfter Sozialleistungen aufweisen können. Mit der Staatsbürgerschaftsreform 2013 wurde die Leistungsbezogenheit insgesamt nochmals unterstrichen und auch auf nicht erwerbsförmige Leistungen ausgeweitet: Erfolgreiche Integration soll nunmehr durch eine beschleunigte Einbürgerung belohnt werden. Neben der Ermessenseinbürgerung nach zehn Jahren, sowie für einige Gruppen (z.B. EU-BürgerInnen und Flüchtlinge) nach sechs Jahren, wird nun ein Einbürgerungsverfahren für „ausgezeichnet Integrierte" (Fast-Track) nach sechs Jahren eingeführt. Die Einstufung „ausgezeichneter Integration" orientiert sich neben den üblichen Einkommensvoraussetzungen hier an höheren Deutschkenntnissen, Beschäftigung im Sozial-, Bildungs- und Gesundheitsbereich, Funktionen in Interessensverbänden, oder der Ableistung ehrenamtlicher Tätigkeiten. Im Kontext der Staatsbürgerschaft wird somit eine weitere Dimension von Leistung sichtbar, in der im Sinne des SSI Integration erwünscht ist: die Ableistung von "Gemeinwohlaktivitäten“.

\subsubsection{Ehrenamtliche Leistungen}

Ehrenamtliches Engagement wird vom SSI als wesentliche Leistungsdimension und als Indikator sozialer Integration präsentiert. Im Rahmen beschleunigter Einbürgerung werden als Beispiele für derartige Leistungen Tätigkeiten im gemeinnützigen Bereich (wie Rotes Kreuz, Caritas, etc.) oder in Interessensvertretungen (Elternvertreter, Betriebsrat, etc.) genannt. Die gesetzliche Öffnung Freiwilliger Feuerwehren für Nicht-StaatsbürgerInnen kommuniziert das SSI als eine Ermöglichung solch gemeinnütziger Leistungen. Freiwilligenarbeit wird zudem über Kampagnen beworben, so etwa in der
Aktion „Zusammen Österreich“ - „Jetzt du! Dein Land braucht dich!", die an Schulen die Mitarbeit in Vereinen und karitativen Organisationen bewirbt. Der Gedanke von Vereinen als der Keimzelle der Integration steht auch hinter den Fördermaßnahmen im Sportbereich, etwa der gesetzlichen Verankerung eines Fördertopfes für Integration im Rahmen der Bundes-Sportförderung oder der Vergabe eines Integrationspreises Sport für Organisationen, welche die Integration von MigrantInnen durch Sport fördern. Nicht zuletzt werden MigrantInnen als MultiplikatorInnen für ihre Communities adressiert, etwa durch Förderprogramme wie das ,MiMiGesundheitslotsInnen-Projekt, in dem MigrantInnen gesundheitsspezifisch geschult werden, um Mitglieder ihrer Sprachgruppe in Gesundheitsfragen ehrenamtlich zu unterstützen.

\subsubsection{Maßnahmen außerhalb des Leistungsprinzips}

Schließlich finden sich unter den integrationspolitischen Maßnahmen der Regierung auch solche, die sich nicht in der Logik einer Leistungsterminologie fassen lassen. Dazu zählt der bereits auf diskursiver Ebene alleinstehende Anspruch an identifikatorische Integration von MigrantInnen, im Sinne eines „Bekenntnisses zur neuen Heimat". Dabei geht es einerseits um die Befolgung rechtlicher Rahmenbedingungen bis hin zu strafrechtlichen Maßnahmen gegen inakzeptable kulturelle Praktiken, z.B. die gesetzliche Verschärfung der Strafverfolgung von Zwangsehen. Andererseits aber auch um habituelle Anpassungen, die eine freiwillige Einordnung des Individuums in den "kulturellen Rahmen“ der neuen Heimat zeigen sollen. Beispiele dafür sind die Einführung einer „Rot-Weiß-Rot-Fibel“ als normativen Orientierungsrahmen für MigrantInnen sowie die Reform des Staatsbürgerschaftstests 20I3, der nun stärker auf „aus der Verfassung ableitbare [...] Grundprinzipien Österreichs" fokussiert ist (BGBL 136/2013). Die Schaffung eines festlichen Verleihungsaktes soll darüber hinaus zur Stärkung eines "Wir"-Gefühls bei Eingebürgerten beitragen und den Wert der Staatsbürgerschaft unterstreichen. $\mathrm{Zu}$ den identifikatorischen Maßnahmen zählen auch spezifisch an MuslimInnen und muslimische Vereine gerichtete Maßnahmen, wie letztlich nicht realisierte Forderungen nach "Deutsch in Moscheen“ oder Maßnahmen des Dialogforum Islam. Dessen Ergebnisse münden in identifikatorische Regelungen im Rahmen der erst in der darauffolgenden Legislaturperiode beschlossenen Novellierung des Islamgesetzes, etwa die Betonung des Vorrangs österreichischen Rechtes vor innerreligiösen Lehren

Auch andere von der Regierung implementierte Integrationsmaßnahmen lassen sich dem Leistungsnarrativ nur unzureichend unterordnen. Dazu gehören gesundheitspolitische Integrationsmaßnahmen wie die Verankerung eines Integrationskoordinators im Haupt- 
verband der österreichischen Sozialversicherungsträger oder wohnpolitische Überlegungen zu einer migrationssensiblen Hausverwaltung bzw. eines desegregierenden Wohnungsmanagements. Hier sind weniger konkrete Leistungserwartungen gegenüber MigrantInnen auszumachen, als vielmehr Maßnahmen, die interkulturelles Zusammenleben sowie soziale Basisversorgung verbessern sollen. Auch die Prämierung interkultureller Vermittlung (etwa durch den ,Journalistenpreis Integration " oder den ,Integrationspreis Sport') lässt sich hier hinzuzählen. Dies verdeutlicht nicht nur die inhaltlichen Grenzen eines Leistungsprinzips für das breite Spektrum integrationspolitischer Maßnahmenbereiche, es wirft auch die Frage auf, in welchem Verhältnis Leistungsdiskurs und integrationspolitische Maßnahmen grundsätzlich stehen?

\subsection{Das Leistungsprinzip zwischen Diskurs und Poli- cy: Kontinuitäten und Brüche}

Die vergleichende Analyse von Integrationsdiskurs und -policies macht deutlich: Vor allem in den zentralen Dimensionen Bildung und Arbeit greifen beide Ebenen nahtlos ineinander. Der narrative Schwerpunkt in diesen Leistungsdimensionen wird durch einen Fokus auf dort $\mathrm{zu}$ verortende politische Maßnahmen eingelöst. Dies entspricht stringent einem auf sozio-ökonomische Integration konzentrierten Integrationsverständnis, kennzeichnen diese Bereiche doch die zentralsten Sozialisationssphären moderner, kapitalistischer Gesellschaftsordnung. Schulbildung und Erwerbstätigkeit umfassen jene Lebensperioden, in welchen von allen Mitgliedern der Gesellschaft individuelle Leistungen als „sozial erwartbar" und damit als einforderbar gelten. Auffällig ist, dass die leistungsbezogenen Maßnahmen keineswegs neu sind. Sowohl im Bildungs- als auch im Arbeitsbereich stellen zahlreiche Maßnahmen eine Fortführung, Ausweitung oder Weiterentwicklung von bereits vorher angelaufenen Maßnahmen dar. Die Innovation des Leistungsfokus in der österreichischen Integrationspolitik besteht darin, eine neue Deutung für bestehende wie für eingeleitete Maßnahmenansätze etabliert zu haben und diese zu einem schlüssigen Ganzen zu verbinden. Daraus folgt, dass den Maßnahmen ihr Leistungsattribut nicht innewohnt, sondern sie diesen Fokus erst im Zuge der (Neu)Deutung durch politische AkteurInnen in diesem Fall eines ÖVP-geführten Staatssekretariats - bekommen. Nicht zuletzt zeigte sich dies auch an wiederholten Spannungen zwischen SPÖ-geführten Ministerien und dem SSI angesichts der Vorstöße des neuen Akteurs, insbesondere im Bildungsbereich. Die Integrationspolitik der Bundesregierung lässt sich daher nur im Spannungsfeld gegensätzlicher ideologischer Motivlagen der Koalitionsparteien adäquat verstehen.
Aus dieser meritokratischen Neudeutung folgt $\mathrm{zu}-$ dem eine zweite Konsequenz, nämlich eine begrenzte Reichweite des Leistungsanspruchs für die Integrationspolitik der Bundesregierung. Am deutlichsten kommt diese Begrenzung in identifikatorischen Integrationsmaßnahmen zum Ausdruck, geht es hier just um solche ethnisch-kulturellen Faktoren, die das Leistungsnarrativ eigentlich zu überwinden vorgibt. Zwar ließen sich derartige Anpassungen in einem weiten Sinne des Begriffs noch als Akkulturationsleistung verstehen, allerdings nicht in dem vom SSI etablierten Narrativ. Darüber hinaus bestehen Maßnahmen, die sich den Ansatzpunkten des Leistungsnarrativs grundsätzlich entziehen. Beispiele sind Maßnahmen im Bereich der Gesundheits- oder Wohnintegration, die weder individuelle Leistung von MigrantInnen einfordern noch sie ermöglichen oder anerkennen, sondern als interkulturelle Vermittlungsangebote bzw. soziale Schutzmaßnahmen angelegt sind.

Dieser begrenzten Reichweite des meritokratischen Narrativs für Maßnahmen jenseits der Bildungs- und Erwerbsintegration wird mit Ausweichstrategien begegnet: Zum einen wird der Leistungsbegriff nicht explizit mit allen integrationspolitischen Forderungen der Bundesregierung verknüpft, sondern es wird in Kauf genommen, dass einzelne Forderungen und Maßnahmen außerhalb dieses Narrativs bleiben. Zum anderen bedient man sich diskursiver Kausalketten, gemäß derer Integrationsleistungen im Bildungs- und Erwerbsbereich in der Folge auch Integration auf sozialer und identifikatorischer Ebene begünstigen: „Österreich-Bewusstsein kann nur entstehen, wenn man erfolgreich ist, sich hier etwas aufbaut, eine Wohnung kauft und Wurzeln schlägt." (Sebastian Kurz in DiePresse, 20IIb). Auf diese Weise bleiben auch darüber hinausgehende Ansprüche letztlich Teil des Leistungsnarrativs, jedoch als dessen Output.

Eine weitere markante Diskrepanz zwischen Diskurs und Policy ergibt sich schließlich mit Blick auf die adressierten AkteurInnen. Während das Leistungsnarrativ noch auf die Beidseitigkeit von Integration, d.h. auf Leistungen sowohl der Mehrheit als auch der Minderheit verweist, zeigt sich bei den geforderten Maßnahmen ein klares Gefälle hin zu Leistungen seitens der „MigrantInnen", denen Rechte und Pflichten direkt zugewiesen werden. „Die Politik“ wird als verantwortliche Instanz der Integrationsförderung angesprochen, vereinzelt auch jene „sozialen Gatekeeper“, die in den betroffenen Politikfeldern für die Umsetzung von Integrationspolitiken verantwortlich sind (z.B. KindergärtnerInnen, LehrerInnen, ArbeitgeberInnen). Die Mehrheitsgesellschaft wird auf Ebene des Narrativs in die Pflicht genommen, in Maßnahmen wird der narrative Anspruch der Beidseitigkeit hingegen nur sehr bedingt eingelöst. Dies spricht dafür, dass der Leistungsdiskurs auch stark als Signal an 
die Mehrheitsgesellschaft dient, eine stärkere Inpflichtnahme von MigrantInnen seitens der Politik sicherzustellen. Damit ist auf die zentrale Frage verwiesen, welche Inklusions- sowie Exklusionspotenziale ein solcher Leistungsturn in der Integrationspolitik für die betroffene Gruppe der MigrantInnen letztlich mit sich bringt?

\section{Inklusions- und Exklusionspotenziale meritokratischer Integrationspolitik}

Analysiert man das Leistungskonzept in der österreichischen Integrationspolitik hinsichtlich inkludierender und exkludierender Effekte, so bringt deren Fokussierung auf "MigrantInnen“ zwei grundsätzliche Probleme mit sich: Zum einen handelt es sich um eine Personengruppe, die neben geringerer Aufenthaltssicherheit auch über weniger soziale und politische Rechte bzw. keine eigenständige politische Stimme (voice) verfügt - Leistungsdruck hat für sie daher gravierendere Konsequenzen als für StaatsbürgerInnen bzw. Daueraufenthaltsberechtigte, v.a. in Zeiten von Wirtschaftskrise, erodierenden Normalarbeitsverhältnissen und sozialstaatlichem Abbau. ${ }^{3}$

Zum anderen befördert dieser Fokus eine Ethnisierung allgemeiner sozialer Problemlagen, die dadurch stärker als Probleme von MigrantInnen denn als gesamtgesellschaftliche Probleme soziostruktureller Benachteiligung wahrgenommen werden (Lutz/Heckmann 20IO; Hormel 2013). Zwar soll die Forderung nach Leistung die Bedeutung der Herkunft einer Person überwinden, ausschließlich an MigrantInnen gerichtet suggeriert sie jedoch einen Kontrast zum - fragwürdigen - Bild einer homogenen, leistenden Mehrheitsgesellschaft. In einem sich verschärfenden Leistungswettbewerb bestehen zu können, wird für MigrantInnen von einer rein wirtschaftlichen Frage somit zum Zugehörigkeitskriterium zur Mehrheitsgesellschaft an sich erklärt.

Neben diesen grundsätzlichen Problemen eines Leistungsansatzes im Integrationskontext lassen sich aber auch fallspezifischere Effekte symbolischer, rechtlicher, ökonomischer und sozialer Inklusion/Exklusion in Österreich hervorheben (Ataç/Rosenberger 2013; Kronauer 20I0; Mourão Permoser 2012). Dem bei Ager und Strang (2008, 18I) beschriebenen Verständnis von Inklusions-/Exklusionsprozessen in der Integrationspolitik folgend, analysieren wir die Leistungsorientierung der Regierungspolitik daher im Hinblick auf die Erweiterung/Verengung von politischen, wirtschaftlichen und gesellschaftlichen Teilhabemöglichkeiten.

3 Die Stratifikation staatsbürgerlicher Rechte basiert auf einer Vielzahl unterschiedlicher Status-Kategorien, mit denen jeweils spezifische bürgerliche, soziale und politische Rechte einher gehen (Ataç/Rosenberger 20I3). Siehe dazu auch die Beiträge von Kai Dröge und Jörg Flecker in diesem Heft.
Der wesentlichste Inklusionszugewinn eines Leistungsansatzes in der Integrationspolitik besteht zweifelsohne in der verstärkten Würdigung - in der Sprache des SSI „Anerkennung" - migrantischer Leistung seitens der Mehrheitsgesellschaft. MigrantInnen werden stärker als bisher in Bezug auf ihren positiven Beitrag zur österreichischen Gesellschaft dargestellt. Positivbeispiele gelungener Integration durch Leistung in Form von sozialen Engagements, v.a. aber aufgrund ökonomischen Erfolgs werden vor den Vorhang geholt und signalisieren MigrantInnen so die Chance zum individuellen Empowerment (aber auch die Vorteile von Diversity in Richtung der Mehrheitsgesellschaft). Diese offensive Betonung sowie die plastische Darstellung in Form von Kampagnen und IntegrationsbotschafterInnen stellt ein Novum in der österreichischen Integrationspolitik und einen klaren Ausdruck symbolischer Inklusion dar.

Ein weiterer Inklusionszugewinn besteht in der partiellen Erweiterung des Leistungsbegriffs, dessen Breite maßgeblichen Einfluss darauf hat, welche gesellschaftlichen Gruppen als leistend oder nicht-leistend betrachtet werden (Degele/Winker 20II). Während ökonomische Leistung und Bildungsleistungen (Sprachkompetenz) weiterhin die zentralen Kriterien für Integration darstellen, wurden sie vorsichtig erweitert: So werden nun auch zivilgesellschaftliche Aktivitäten wie Ehrenamt und Tätigkeiten in Interessensvertretungen als integrative Leistung betrachtet und erwünscht. Derartige Ausweitungen des Leistungsspektrums gelten in der Meritokratieforschung als inklusionsfördernd, sofern damit weitere Möglichkeiten eröffnet und nicht zusätzliche Verpflichtungen auferlegt werden (McNamee/Miller 2004). Dieses Potenzial könnte durch einen umfassenderen Leistungsbegriff noch extensiver ausgeschöpft werden, der etwa auch weitere nicht-erwerbsförmige Leistungen berücksichtigt.

Exklusionseffekte bestehen durch die fortgeführte Konditionalität von Aufenthaltsstatus und damit verbundenen Rechten: Im Unterschied zu StaatsbürgerInnen und Daueraufenthaltsberechtigten stehen Neuzuwanderer oder befristet Aufenthaltsberechtigte vor der Herausforderung, zuerst dem Leistungsanspruch genügen zu müssen, bevor ihnen vergleichbare rechtliche Teilhabechancen gewährt werden. Dies widerspricht jenen Konzepten gesellschaftlicher Inklusion, die Rechte als notwendige Basis jedes Integrationsprozesses verstehen (Marshall I950; Ager/Strang 2008; Kronauer 20IO; Ataç/Rosenberger 20I3). Konsequenzen eines solchen Zugangs zeigen sich etwa im rechtlichen Ausschluss einkommensschwacher Personen von Staatsbürgerschaft und damit politischer Teilhabe (Stadlmair 20I5; Stern 20I2), der sich im Sozialprofil eingebürgerter Personen niederschlägt (Reichel/Perchinig 2014).

Ambivalent bleibt schließlich auch der Befund zu Anti-Diskriminierung: Egalitaristischen Verständnis- 
sen von Meritokratie wohnt der Anspruch inne, ungleiche Startbedingungen von Menschen aufgrund individueller sozio-ökonomischer Hintergründe und Lebensumstände auszugleichen (Roemer 1998). Mit Fördermaßnahmen für MigrantInnen in den Bereichen Bildung und Arbeit setzt die Bundesregierung wesentliche Schritte, um solchen Ausgangsdefiziten zu begegnen, was zunächst als inklusive Politikentwicklung zu werten ist. Demgegenüber bleibt der exkludierende Effekt allgemeiner struktureller Hürden (Bruneforth/Lassnig 20I2) sowie diskriminierender Selektionspraktiken (Bacchi 1999) im Bildungs- und Arbeitsmarktsystem aber weitgehend bestehen. Fehlt der Ausbau solcher Anti-Diskriminierungsmaßnahmen und affirmative action policies, so bleibt "Leistungsgerechtigkeit" lediglich ein Etikett und versperrt den Blick auf dahinter liegende Ursachen sozialer Selektion und Segmentierung.

\section{Schlussfolgerungen}

Mit der Institutionalisierung der österreichischen Integrationspolitik auf Bundesebene durch das Staatssekretariat für Integration ist eine meritokratische Neuausrichtung verbunden, die Fragen nach der Tragfähigkeit eines solchen Ansatzes im Politikfeld Integration aufwirft.

Der vorliegende Beitrag macht erstens deutlich, dass es sich dabei vor allem um eine diskursive Neudeutung handelt: Durch das Narrativ „Integration durch Leistung" wird Integration als eine gestaltbare gesellschaftliche Herausforderung erfasst, mit einem Fokus auf Sprache, Bildung und Arbeit sowie mit spezifischen Aufgaben für Minderheit, Mehrheit und Politik. Dieser diskursiven Neuausrichtung des Regierungsdiskurses steht auf Policy-Ebene jedoch eine Kontinuität integrationspolitischer Maßnahmenfelder (von Sprachförderung, über Bildungsintegration bis Arbeitsmarktqualifizierung) gegenüber, welche auch schon davor - wenngleich nicht unter einem Leistungsnarrativ kommuniziert - zu finden waren. Leistung stellt somit kein neues Kriterium von Integrationspolitik dar, sondern in erster Linie eine neue Perspektive, die Migration und Integration abseits kulturalistischer Zugänge betrachtet.

Zweitens zeigt der Beitrag grundlegende Spannungsmomente aus der Verknüpfung integrationspolitischer Herausforderungen mit meritokratischen Lösungen auf, in denen sich Grundfragen der Meritokratieforschung nach dem Spannungsverhältnis von Meritokratie und demokratischem Gleichheitspostulat (Rawls 1972; Young 1990; Sen 1999) widerspiegeln:

So fördert die Anwendung eines individualistischen Prinzips (Leistung) in einem gruppenzentrierten Politikfeld (MigrantInnen) den Konflikt zwischen der proklamierten Irrelevanz von Herkunft und Leistungsansprüchen an eine permanent durch gerade diese Her- kunft (bzw. ihren Aufenthaltsstatus) identifizierte Gruppe. Dies trägt zur Ethnisierung gesamtgesellschaftlicher sozio-struktureller Problemlagen bei und setzt zugleich der Durchgängigkeit des Leistungsprinzips in der Integrationspolitik Grenzen. Ihnen wäre letztlich erst dadurch zu begegnen, dass meritokratische Integrationspolitik nicht mehr gruppenspezifisch agiert, sondern die Integration aller Nicht-Leistenden in Form von Mainstreaming fördert (Collett/Petrovic 20I4). Das Fallbeispiel Österreich zeigt hingegen ein Nebeneinander von Leistungsansatz sowie herkunfts- und kulturbasierten Zugängen in einer auf MigrantInnen fokussierten Integrationspolitk. Gerade bei diesem Vorgehen können soziale, ökonomische und politische Inklusion nur dann realisiert werden, wenn auch Anti-Diskriminierungsmaßnahmen und Affirmative Action noch zentralere Bausteine als bisher darstellen, um nicht-meritokratisch begründete Benachteiligungen zu eliminieren und zu effektiver Gleichheit von Leistungschancen sowie Leistungsgratifikation beizutragen.

Letztlich sind diese Befunde jedoch stets vor der grundsätzlichen Frage zu sehen, inwieweit Leistungsgesellschaft und meritokratische Politik überhaupt die gesellschaftliche Integrität zu stärken vermögen oder sie vielmehr gefährden. Zwar ist die Hinwendung zu Leistung und Leistungswettbewerb heute ein weithin beobachtbares politisches Phänomen in zahlreichen Politikfeldern, jedoch vielfach mit exkludierenden Konsequenzen für strukturell benachteiligte Bevölkerungsgruppen, v.a. solche ohne eigene politische Stimme (siehe dazu die übrigen Beiträge dieses Themenheftes). In jenem Maße, in dem Integrationspolitik sich an diesen Politikfeldern orientiert - wie am Fallbeispiel Österreich der Fokus auf Bildung und Arbeit zeigt -, werden auch die dortigen Probleme des Leistungsprinzips mitübernommen und dessen immanente Grenzen offensichtlich.

\section{Literatur}

Ager, Alastair/Alison Strang (2008). Understanding Integration. A Conceptual Framework, in: Journal of Refugee Studies, Vol. 2 I(2), I66-I9I.

Ataç, Ilker/Sieglinde Rosenberger (2013). Inklusion/Exklusion - ein relationales Konzept der Migrationsforschung, in: Ataç, Ilker/Sieglinde Rosenberger (Hg.): Politik der Inklusion und Exklusion, Wien, 35-52.

Bauböck, Rainer (1996). „Nach Rasse und Sprache verschieden“. Migrationspolitik in Österreich von der Monarchie bis heute, in: Reihe Politikwissenschaft 3I, Institut für Höhere Studien, Wien. Internet: https://www.ihs.ac.at/publications/pol/pw_3I.pdf (Zugriff: 12.3.2010). 
Bemelmans-Videc, Marie-Louise/Ray C. Rist/Evert Vedung (Hg.) (20IO). Carrots, Sticks \& Sermons. Policy Instruments and Their Evaluation, New Brunswick (NJ).

Bischoff, Joachim (2005). Das Ende des Neoliberalismus und die Zukunft der Wirtschaftsdemokratie, in: UTOPIE kreativ, Vol. I73, 200-2II.

Boswell, Christina/Andrew Geddes/Peter Scholten (2011). The Role of Narratives in Migration Policy-Making: A Research Framework, in: The British Journal of Politics \& International Relations, Vol. I3(I), I-II.

Bruneforth, Michael/Lorenz Lassnig (2012). Nationaler Bildungsbericht Österreich 20I2. Band I: Das Schulsystem im Spiegel von Daten und Indikatoren, Graz. Internet: https://www.bifie.at/system/files/buch/ pdf/NBB2OI2_Band\%2OI_gesamt.pdf (Zugriff: I2.3.2016)

Castilla, Emilio J. (2008). Gender, Race, and Meritocracy in Organizational Careers, in: American Journal of Sociology, Vol. II3(6), I479-I526.

Collett, Elizabeth/Milica Petrovic (2014). The Future of Immigrant Integration in Europe: Mainstreaming Approaches for Inclusion, Migration Policy Institute, Brüssel. Internet: http://migrationpolicy.org/ research/future-immigrant-integration-europemainstreaming-approaches-inclusion (Zugriff: I2 March 2015).

Degele, Nina/Gabriele Winker (2OII). 'Leistung muss sich wieder lohnen'. Zur intersektionalen Analyse kultureller Symbole, in: Katharina Knüttel/Martin Seeliger (Hg.): Intersektionalität und Kulturindustrie. Zum Verhältnis sozialer Kategorien und kultureller Repräsentationen, Bielefeld, 25-53.

Der Standard (2012). Wir müssen eine Willkommenskultur entstehen lassen, in: derstandard.at, I0.8.2012. Internet: http://derstandard.at/1343744545422/Wirmuessen-eine-Willkommenskultur-entstehen-lassen (Zugriff: 9.9.20I3).

Der Standard (2OI3): „Fernhalten, nicht einbürgern“, in: derstandard.at, 6.2.2013. Internet: http://derstandard.at/1360I6095697I/Fernhalten-nicht-einbuergern (Zugriff: 9.9.2013).

Die Presse (20Ira). Diskussion: „Junge Türken fühlen sich nicht zu Hause", in: DiePresse.com, 9.7.20II. Internet: http://diepresse.com/home/politik/innenpolitik/676587/Diskussion_Junge-Turken-fuhlen-sichnicht-zu-Hause (Zugriff: 24.8.2015).

Die Presse (20Irb): „Kurz: „Viele Migranten befinden sich in einer Identitätskrise «", in: DiePresse.com, 3.I2.2OII. Internet: http://diepresse.com/home/politik/innenpolitik/7I4085/Kurz_Viele-Migrantenbefinden-sich-in-einer-Identitaetskrise (Zugriff: I2.5.20I2).

Die Presse (2013). Sebastian Kurz: Gut integriert in Wien?, in: DiePresse.com, 20.4.2013. Internet: http:// diepresse.com/home/politik/innenpolitik/I39I837/
Sebastian-Kurz_Gut-integriert-in-Wien (Zugriff: I3.8.2015).

Foucault, Michel (1994). Überwachen und Strafen. Die Geburt des Gefängnisses, Frankfurt.

French, John R./Bertram Raven (1959). The Bases of Social Power, in: Dorwin Cartwright (Hg.): Studies in Social Power, Ann Arbor, MI, 150-I67.

Goodman, Sara Wallace (2010). Integration Requirements for Integration's Sake? Identifying, Categorising and Comparing Civic Integration Policies, in: Journal of Ethnic and Migration Studies, Vol. 36(5), 753-772.

Goodman, Sara Wallace (2OI4). Immigration and Membership Politics in Western Europe, Cambridge.

Götzelmann, Andrea (20I0). Die Rolle staatlicher AkteurInnen in der österreichischen Integrationspolitik', in: Herbert Langthaler (Hg.): Integration in Österreich. Sozialwissenschaftliche Befunde, Innsbruck, I8I208.

Gruber, Oliver (20I4). Campaigning in Radical Right Heartland. The electoral politicization of immigration and ethnic relations in Austrian general elections, I97I-2013, Münster.

Gruber, Oliver/Astrid Mattes (2015). Das Narrativ ,Integration durch Leistung'. Neuansatz staatlicher Integrationspolitik?, in: Karin B. Schnebel (Hg.): Europäische Minderheiten. Im Dilemma zwischen Selbstbestimmung und Integration, Wiesbaden, 89-II6.

Joppke, Christian (2007). Beyond national models: Civic integration policies for immigrants in Western Europe, in: West European Politics, Vol. 30(I), I-22.

Jorgensen, Martin Bak/Trine Lund Thomsen (2013). "Needed but Undeserving" - Revisiting the Liberal Paradox. Internet: http://www.recode.info/ wp-content/uploads/2OI/oI/Final-RECODE32-j\%C3\%B8rgensen-and-thomsen_Final_fin.pdf (Zugriff: 31.8. 2014).

Kaplan, Thomas J. (1986). The Narrative Structure of Policy Analysis, in: Journal of Policy Analysis and Management, Vol. 5(4), 76I-778.

Kolb, Holger (2008). States as Clubs? The Political Economy of State Membership, in: Holger Kolb/Henrik Ekberg (Hg.): Migrants and Markets. Perspectives from Economics and Other Social Sciences, Amsterdam, I2O-I 47.

König, Karin (1998). Das österreichische Migrationsregime von 1945 bis heute, in: Vor der Information (Sonderheft: „Staatsarchitektur”), Vol. 7(8), I5O-I55.

Koopmans, Ruud (2002). Codebook for the analysis of political mobilisation and communication in European public spheres. Internet: http://europub.wzb.eu/ Data/Codebooks\%2oquestionnaires/D2-I-claimscodebook.pdf (Zugriff: 27.3.20I3).

Kraler, Albert (20II). Immigrant and Immigration Policy Making in Austria, in: Giovana Zincone/Rinus Penninx/Maren Borkert (Hg.): The Making of Migration 
and Integration Policies in Europe. Processes, Actors and Contexts in Past and Present, Amsterdam, 2I-59.

Kronauer, Martin (20I0). Exklusion. Die Gefährdung des Sozialen im hoch entwickelten Kapitalismus, 2.Aufl., Frankfurt.

Krzyzanowski, Michal/Ruth Wodak (2008). The Politics of Exclusion: Debating Migration in Austria, New Brunswick, NJ.

Kunz, Thomas (20II). Integration fördern und fordern, in: Sigrid K. Amos/Wolfgang Meseth/Matthias Proske (Hg.): Öffentliche Erziehung revisited, Wiesbaden, 323-344.

Lahusen, Christian/Carsten Stark (2003). Integration: Vom fördernden und fordernden Wohlfahrtsstaat, in: Stephan Lessenich (Hg.): Wohlfahrtsstaatliche Grundbegriffe. Historische und aktuelle Diskurse, Frankfurt, 353-372.

Lanz, Stephan (2009). In unternehmerische Subjekte investieren. Integrationskonzepte im Workfare-Staat. Das Beispiel Berlin, in: Sabine Hess/Jana Binder/Johannes Moser (Hg.): No integration?! Kulturwissenschaftliche Beiträge zur Integrationsdebatte in Europa, Bielfeld, I05-I22.

Lutz, Anna/Friedrich Heckmann (20IO). Die Bevölkerung mit Migrationshintergrund in Bayern. Stand der Integration und integrationspolitische Maßnahmen, Bamberg.

Massey, Eric/Dave Huitema (2013). The emergence of climate change adaptation as a policy field: the case of England, in: Regional Environmental Change, Vol. I3(2), 34I-352.

McNamee, Stephen J./Robert K. Miller (2004). The Meritocracy Myth, in: Sociation Today, Vol. 2(I). Internet: http://www.ncsociology.org/sociationtoday/v2 I/merit.htm (Zugriff: I4.3.20I6).

Michalowski, Ines/Ricky van Oers (2012). How Can We Categorise and Interpret Civic Integration Policies?, in: Journal of Ethnic and Migration Studies, Vol. 38(I), I63-I7I.

Moore, R. Quinn (2000). Multiracialism and Meritocracy: Singapore's Approach to Race and Inequality, in: Review of Social Economy, Vol. 58(3), 339-36o.

Mourão Permoser, Julia (2012). Civic Integration as Symbolic Politics: Insights from Austria, in: European Journal of Migration and Law, Vol. I4, I73-I98.

Mourão Permoser, Julia/Sieglinde Rosenberger (20I2). Integration Policy in Austria, in: Frideres, James/John Biles (Hg.): International Perspectives: Integration and Inclusion, Montreal, 39-58.

Mouritsen, Per (20I3). The Resilience of Citizenship Traditions: Civic Integration in Germany, Great Britain and Denmark, in: Ethnicities, Vol. I3(I), 86-Io9.

Oliver, Pamela (1980). Rewards and Punishments as Selective Incentives for Collective Action: Theoretical
Investigations, in: The American Journal of Sociology, Vol. 85(6), I356-I375.

Opielka, Michael (Hg.) (2005). Bildungsreform als Sozialreform. Zum Zusammenhang von Bildungs- und Sozialpolitik, Wiesbaden.

OTS (20IO). Fekter: Sprachkenntnisse sind der Schlüssel für erfolgreiche Integration. APA-OTS, Bundesministerium für Inneres, I9.IO.20IO. Internet: http://www. ots.at/presseaussendung/OTS_2OIOOII9_OTSOI58/ fekter-sprachkenntnisse-sind-der-schluessel-fuererfolgreiche-integration (Zugriff: 27.I.2010).

OTS (20II). Kopf: Brauchen Leistung für Wohlstand und Solidarität. APA-OTS, ÖVP-Parlamentsklub, I5.9.2OII. Internet: http://www.ots.at/presseaussendung/OTS_2OIIO9I5_OTSO269/kopf-brauchenleistung-fuer-wohlstand-und-solidaritaet (Zugriff: IO.9.2OI3).

OTS (20I2a). Kurz: Migranten zur Freiwilligkeit motivieren; ab heute darf jeder Migrant zur Freiwilligen Feuerwehr. APA-OTS, Staatssekretariat für Integration, 8.3.20I2. Internet: http://www.ots.at/presseaussendung/OTS_2OI20308_OTSO236/kurz-migranten-zur-freiwilligkeit-motivieren-ab-heute-darfjeder-migrant-zur-freiwilligen-feuerwehr (Zugriff: II.9.2OI3).

OTS (2012b). „Integration im Klassenzimmer": Zwischen Herausforderung und Chance. APA-OTS, Österreichischer Integrationsfonds, I7.2.20I2. Internet: http://www.ots.at/presseaussendung/OTS_2OI2 O2I7_OTSoo64/integration-im-klassenzimmerzwischen-herausforderung-und-chance (Zugriff: 24.8.20I5).

OTS (20I2c). LH Pröll und StS Kurz ziehen bei sprachlicher Frühförderung an einem Strang. APA-OTS, Staatssekretariat für Integration, 5.4.20I2. Internet: http://www.ots.at/presseaussendung/ OTS_2OI20405_OTSOO45/lh-proell-und-sts-kurzziehen-bei-sprachlicher-fruehfoerderung-an-einem-strang (Zugriff: 24.8.2015).

Pelinka, Anton/Sieglinde Rosenberger (2007). Österreichische Politik. Grundlagen - Strukturen - Trends, Vienna.

Perchinig, Bernhard (2010). Ein langsamer Weg nach Europa. Österreichische (Arbeits)migrations- und Integrationspolitik seit 1945, in: Leibnitz Institut für Sozialwissenschaften/Bundesamt für Migration und Flüchtlinge (Hg.): Migration und ethnische Minderheiten, Mannheim, II-32.

Perchinig, Bernhard(2012). The national policy frames for the integration of newcomers, ICMPD, Wien. Internet: http://research.icmpd.org/fileadmin/ResearchWebsite/Project_material/PROSINT/Reports/WP2_ CompRep_Finalo30520I2.pdf (Zugriff: I5.OI.20I6).

Rawls, John (1972). A Theory of Justice, Oxford. 
Reichel, David/Bernhard Perchinig (20I4). Reflections on the Value of Citizenship - Explaining Naturalisation Practices, in: Österreichische Zeitschrift für Politikwissenschaft, Vol. 44(I), 32-45.

Rieger, Günther (2005). Meritokratie, in: Nohlen, Dieter/ Rainer-Olaf Schultze (Hg.): Lexikon der Politikwissenschaft, 3. Aufl., München, 565.

Roemer, John E. (1998). Equality of Opportunity, in: Kenneth Arrow/Samuel Bowles/Steven Durlauf (Hg.): Meritocracy and economic inequality, Princeton, 17-32.

Segert, Astrid (2008). Zur Bedeutung von Ungleichheitssemantiken für die Erwerbsintegration von MigrantInnen. Internet: http://irihs.ihs.ac.at (Zugriff: 23.5.2015).

Sen, Amartya (1999). Merit and Justice, in: Kenneth Arrow/Samuel Bowles/Steven Durlauf(Hg.): Meritocracy and Economic Inequality, Princeton, 5-I6.

Shanahan, Elizabeth A./Michael D. Jones/Mark K. McBeth (20II). Policy Narratives and Policy Processes, in: Policy Studies Journal, Vol. 39(3), 535-561.

Staatssekretariat für Integration (20II). Leitbild. Internet: http://www.integration.at/wir_ueber_uns/ ( $\mathrm{Zu}-$ griff: 12.9.2013).

Stadlmair, Jeremias (2015). Ökonomische Einbürgerungsvoraussetzungen im europäischen Vergleich: Irland, Dänemark und Österreich, in: Karin B. Schnebel (Hg.): Europäische Minderheiten. Im Dilemma zwischen Selbstbestimmung und Integration, Wiesbaden, 165200.

Stern, Joachim (2012). Ius Pecuniae - Staatsbürgerschaft zwischen ausreichendem Lebensunterhalt, Mindestsicherung und Menschenwürde, in: Julia Dahlvik (Hg.): Migration und Integration, Göttingen, 55-73.

Stern, Joachim/Gerd Valchars (2013). Austria, EUDO Citizenship Observatory, San Domenico di Fiesole. Internet: http://eudo-citizenship.eu/admin/?p=file\&ap $\mathrm{pl}=$ countryProfiles $\& \mathrm{f}=2013-28$-Austria.pdf (Zugriff: I0.5.2014).

Strasser, Sabine/Jelena Tošić (2013). Egalität, Autonomie und Integration: Post-Multikulturalismus in Österreich, in: Boris Nieswand/Heike Drotbohm (Hg.): Kultur, Gesellschaft, Migration. Die reflexive Wende in der Migrationsforschung, Wiesbaden, I23-150.

Volf, Patrick/Rainer Bauböck (Hg.) (200I). Wege zur Integration. Was man gegen Diskriminierung und Fremdenfeindlichkeit tun kann, Klagenfurt.

Young, Iris M. (1990). Justice and the Politics of Difference, Princeton, NY.

\section{AutorInnen}

Oliver Gruber, geb. 198I, ist Projektmitarbeiter und Universitätslektor für Politikwissenschaft an den Universitäten Wien und Innsbruck sowie Mitglied der
Forschungsgruppe „IN:EX Politics of Inclusion and Exclusion“. Zu seinen Forschungsschwerpunkten zählen u.a. Migrations- und Integrationspolitik, Parteien und Politisierung sowie Demokratische Inklusion und Österreichische Politik. Zuletzt erschienen: The End of the Affair: A Comparative Study of How Party Leadership Terms End. In: William Cross and Jean-Benoit Pilet (Eds.) The Politics of Party Leadership: A Cross-National Perspective. Oxford University Press, 2015, I28-I48 (mit Scott Pruysers, William Cross, Tim Bale); And it's good night Vienna. How (not) to deal with the populist radical right: The conservatives, UKIP and some lessons from the heartland. In: British Politics, 9(3), 237-254 (mit Tim Bale); Campaigning in Radical Right Heartland. The electoral politicization of immigration and ethnic relations in Austrian general elections, I97I-20I3. LIT Verlag, 2014.

Astrid Mattes, geb. 1988, ist Universitätsassistentin am Institut für Politikwissenschaft der Universität Wien. Migration/Integration und religiöse Diversität in Europa sind Forschungsschwerpunkte der Politik- und Religionswissenschaftlerin. Als Mitglied der Forschungsgruppe „IN:EX Politics of Inclusion and Exclusion“ steht dabei die kritische Auseinandersetzung mit gesellschaftlichen Exklusionsmechanismen und Inklusionspotenzialen im Zentrum. Aktuelle Publikationen: Institutional Responses to Religious Diversity", Interdisciplinary Journal for Religion and Transformation in Contemporary Society, 2016 (I) (with Kristina Stoeckl and Julia Mourao Permoser); "Towards a Universal Religion? Symbolic Boundaries in Austrian Immigrant Integration Policies" In: Appel, Kurt; Guanzini, Isabella (ed.): Rethinking Europe with(out) Religion III. V\&R Unipress. 2015, 219-240.

Jeremias Stadlmair, geb. 1987, ist Universitätsassistent und Dissertant am Institut für Politikwissenschaft der Universität Wien, sowie Mitglied der Forschungsgruppe „IN:EX Politics of Inclusion and Exclusion“. Seine Forschungsgebiete umfassen Migrations- und Staatsbürgerschaftspolitik, politische Partizipation und direkte Demokratie. Zuletzt erschienen: „Direkte Demokratie - Regierungstechnik oder Instrument der BürgerInnen?" in: Öhlinger, Theo/Poier, Klaus (Hrsg.): Direkte Demokratie und Parlamentarismus. Wie kommen wir zu den besten Entscheidungen? Böhlau. 2015, 227-25I (mit Sieglinde Rosenberger); „Ökonomische Einbürgerungsvoraussetzungen im europäischen Vergleich: Irland, Dänemark und Österreich“ in: Schnebel, Karin (Hrsg.): Europäische Minderheiten. Im Dilemma zwischen Selbstbestimmung und Integration. VS Verlag für Sozialwissenschaften. 2014, 165-200. 
